\title{
New Scenario of plasma evolution in IC 443
}

\author{
Arisa Hirayama ${ }^{1}{ }^{*}$, Shigeo Yamauch ${ }^{1}$, Kumiko K. Nobukawa ${ }^{1}$, \\ Masayoshi NobUKaWA ${ }^{2}$, and Katsuji Koyama ${ }^{3}$ \\ ${ }^{1}$ Department of Physics, Nara Women's University, Kitauoyanishimachi, Nara 630-8506 \\ ${ }^{2}$ Faculty of Education, Nara University of Education, Takabatake-cho, Nara 630-8528 \\ ${ }^{3}$ Department of Physics, Graduate School of Science, Kyoto University, \\ Kitashirakawa-oiwake-cho, Sakyo-ku, Kyoto 606-8502 \\ *E-mail: raa-hirayama@cc.nara-wu.ac.jp \\ Received; Accepted
}

\begin{abstract}
Most of young and middle-aged supernova remnants (SNRs) exhibit an ionizing plasma (IP), an ionizing process following a shock heated SNR gas. On the other hand, significant fractions of SNRs exhibit a recombining plasma (RP). The origin and the mechanisms of the RP, however, are not yet well understood. This paper proposes a new model that the RP is followed after the IP process taken at the first epoch of the SNR evolution. Using the high quality and wide band $(0.6-10 \mathrm{keV})$ spectrum of IC 443 , we nicely fitted with a model of two RP (two-RP model) plus a power law (PL) with an FeI K $\alpha$ line component. The ionization temperature in one RP monotonously increases from $\mathrm{Ne}-\mathrm{Ca}$, while that in the other RP shows a drastic increase from $\mathrm{Cr}-\mathrm{Ni}$. Origin and mechanism of the two- $\mathrm{RP}$ and $\mathrm{PL}$ with an $\mathrm{Fe}$ I $\mathrm{K} \alpha$ line components are possibly due to a different evolution of two plasmas and ionization by the low-energy cosmic ray.
\end{abstract}

Key words: ISM: individual (IC 443) — ISM: supernova remnants - X-rays: ISM 


\section{Introduction}

The common concept of a shock heated plasma of supernova remnants (SNRs) is that electron temperature $\left(k T_{\mathrm{e}}\right)$ and ionization temperature $\left(k T_{\mathrm{i}}\right)$ are nearly $\sim 0 \mathrm{keV}$ (atoms are nearly neutral) at the initial epoch. Soon after, $k T_{\mathrm{e}}$ increases quickly, and then gradually ionized atoms, or $k T_{\mathrm{i}}$ follows after $k T_{\mathrm{e}}$. Thus the SNR plasma is not in collisional ionization equilibrium (CIE: $k T_{\mathrm{e}}=k T_{\mathrm{i}}$ ), but is an ionizing plasma (IP: $k T_{\mathrm{e}}>k T_{\mathrm{i}}$ ). Since X-ray spectra of most of the young-middle aged SNRs are well fitted with the IP model, IP is widely accepted to be a standard SNR evolution (hereafter, IP SNR). However, recently some SNRs have been known to exhibit a recombining plasma (RP) of $k T_{\mathrm{e}}<k T_{\mathrm{i}}$. (hereafter, RP SNR). On the contrary to the clear physics of IP SNRs, physics for the evolution of RP SNRs or how to make $k T_{\mathrm{e}}<k T_{\mathrm{i}}$ plasma has been so far unclear.

IC $443(\mathrm{G} 189.1+3.0)$ is an SNR with the diameter of $45^{\prime}$ at the distance of $1.5 \mathrm{kpc}$ (e.g., Welsh \& Sallmen 2003). In the X-ray band, the age is estimated to be 3000-30000 yr (e.g., Petre et al. 1988; Olbert et al. 2001), a middle-old aged SNR. It is also reported as a core collapsed (CC-SNR) and mixed morphology SNR (MM-SNR: Rho \& Petre 1998). A hint of RP was discovered in the IC

443 spectrum for the first time by Kawasaki et al. (2002). Yamaguchi et al. (2009) then discovered an enhanced structure of the radiative recombining continuum (RRC) of the He-like silicon (Si XIII) and sulfur (SXV), which were direct evidence for RP. The RRC of the He-like iron (Fe XXV), a key element of the evolution, was found from this middle-old aged SNR in the limited energy band of 3.7$10 \mathrm{keV}$ (Ohnishi et al. 2014). The RRC structures of these key elements play a key roll to investigate the spectral evolution of the RP SNRs. We, therefore, made a deep observation of IC 443 with Suzaku (Mitsuda et al. 2007) to establish the RP structures in many key elements.

Conventionally, a model of the RP process is treated to start from a common $k T_{\mathrm{i}}$ in all the elements. We propose a new model of the RP, which starts from element-dependent $k T_{\mathrm{i}}(z)$. For the verification of this new model, and to combine the RP to the well-established IP process, we utilize the $0.6-10 \mathrm{keV}$ band spectrum of IC 443 obtained in the $\sim 400 \mathrm{ks}$ exposure observations with Suzaku. Details of the new RP model, analysis process and results are presented in section 3. Based on the results, implications for the RP origin in IC 443 are discussed in section 4. The quoted errors are in the $90 \%$ confidence limits.

\section{Observations}

The Suzaku observations of IC 443 were performed with the X-ray Imaging Spectrometer (XIS: Koyama et al. 2007) placed at the focal planes of the thin foil X-ray Telescopes (XRT: Serlemitsos et al. 2007). The XIS consisted of 4 sensors. One of the XIS (XIS1) is a back-side illuminated CCD 
(BI), while the other three XIS sensors (XIS0, 2, and 3) are a front-side illuminated CCD (FI). In order to achieve the highest count rate ratio of IC 443/NXB in the higher energy band, we only used the FI following Ohnishi et al. (2014). The count rate ratio of IC 443/NXB is determined by the count rates of IC 443 and NXB. The photon count rate ratio of FI and BI of IC 443 is estimated by their quantum efficiencies, and is $\sim 1.5-1.8$ at the Fe XXVI Ly $\alpha$ and RRC energy band of 7-10 keV (see Fig. 4 of Koyama et al. 2007), while the count rate ratio of NXB is $\sim 0.3-0.06$ (see Fig. 16 of Koyama et al. 2007). As a matter of fact, the observed IC 443 photon count rates of FI and BI are $3.0 \times 10^{-3}$ counts $\mathrm{s}^{-1}$ and $1.5 \times 10^{-3}$ counts $\mathrm{s}^{-1}$, while the count rates for $\mathrm{NXB}$ are $1.56 \times 10^{-2}$ counts $\mathrm{s}^{-1}$ and $6.44 \times 10^{-2}$ counts $\mathrm{s}^{-1}$, respectively. Therefore, the count rate ratio of IC $443 / \mathrm{NXB}$ of FI is $\sim 0.2$, about 10 times better than that of BI of $\sim 0.02$. Accordingly, we only use the FI in the analysis of the high energy band of 7-10 keV. The worse count rate ratio of IC 443/NXB of BI than FI is mainly attributable to the larger NXB count rate of BI than FI. The data used in this paper are listed in Table 1.

The XIS was operated in the normal clocking mode. The field of view (FOV) of the XIS is $17 ! 8 \times 17 ! 8$. The XIS employed the spaced-row charge injection (SCI) technique to recover the spectral resolution (Uchiyama et al. 2009). The XIS data in the South Atlantic Anomaly, during the earth occultation, and at the low elevation angle from the earth rim of $<5^{\circ}$ (night earth) and $<20^{\circ}$ (day earth) are excluded. Removing hot and flickering pixels, the data of the Grade 0, 2, 3, 4, and 6 are used. The XIS pulse-height data are converted to Pulse Invariant (PI) channels using the xispi software in the HEAsoft 6.19 and the calibration database version 2016-06-07. We used only XIS0 and XIS3 after 2006 November because XIS2 became out-of-function after the epoch. A small fraction of the XIS0 area became unavailable, possibly due to an impact of micro-meteorite on 2009 June 23. After the epoch, we ignore the damaged area of the XIS0.

\section{Analysis and Results}

\subsection{X-ray Image}

X-ray photons of IC 443 are taken from the data in the observations listed in table 1 (1-5 rows). After subtraction of the non X-ray background (NXB) generated by xisnxbgen (Tawa et al. 2008), the X-ray images in the 2.2-5.2 keV (color map) and 5.5-10 keV (green contour) bands are shown in figure 1, where calibration source regions are excluded. These energy bands are selected from ejecta dominant plasma (see figures $3 \mathrm{c}$ and $3 \mathrm{~d}$ ). In comparison with diffuse structure in the 2.2-5.2 keV band, the 5.5-10 keV band image shows clear concentration toward the image center (cross mark). This contrast would be closely related to the 2-component spectral structure found in section 3.4. 
Table 1. Observation logs.

\begin{tabular}{|c|c|c|c|}
\hline Obs. ID & $\begin{array}{c}\text { Obs. date } \\
\text { start time - end time }\end{array}$ & (R.A., Dec.) J2000.0 & $\begin{array}{c}\text { Exposure } \\
(\mathrm{ks})\end{array}$ \\
\hline \multicolumn{4}{|c|}{ IC 443} \\
\hline 501006010 & 2007-03-06 10:40:19 - 2007-03-07 12:22:14 & $(6: 17: 11,22: 46: 32)$ & 42.0 \\
\hline 507015010 & 2012-09-27 05:29:48 - 2012-09-29 18:40:22 & $(6: 17: 11,22: 45: 12)$ & 101.8 \\
\hline 507015020 & 2013-03-27 04:15:06 - 2013-03-28 16:00:19 & $(6: 17: 12,22: 44: 47)$ & 59.3 \\
\hline 507015030 & 2013-03-31 11:44:34 - 2013-04-03 21:12:21 & $(6: 17: 12,22: 44: 46)$ & 131.2 \\
\hline 507015040 & 2013-04-06 05:21:49 - 2013-04-08 02:00:21 & $(6: 17: 12,22: 44: 52)$ & 75.6 \\
\hline \multicolumn{4}{|c|}{ Background } \\
\hline 409019010 & 2014-10-05 15:12:56 - 2014-10-07 19:09:17 & $(6: 27: 15,14: 53: 24)$ & 82.1 \\
\hline
\end{tabular}

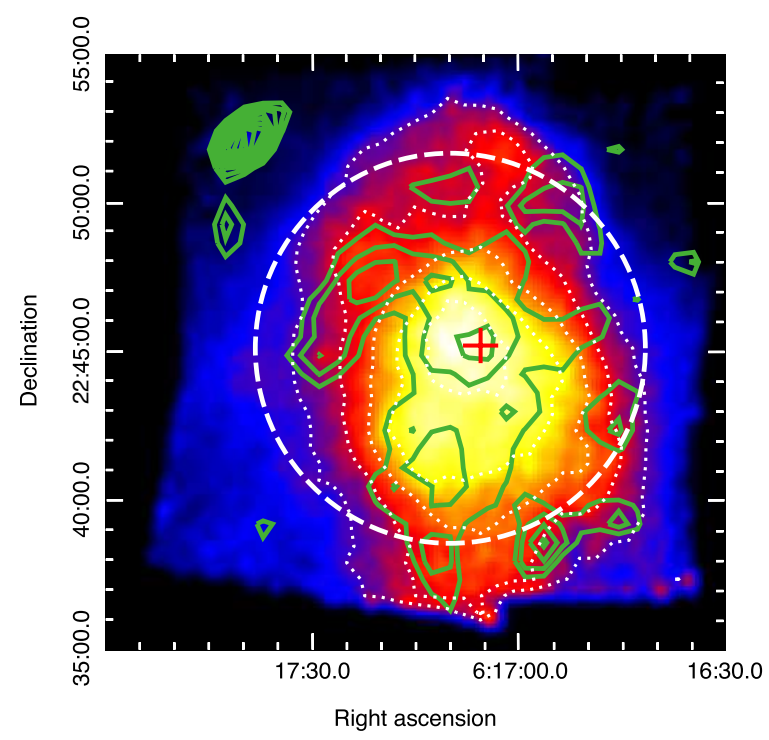

Fig. 1. X-ray map of the main part of IC 443 in the $2.2-5.2 \mathrm{keV}$ (color image and white dotted line, RP1) and 5.5-10 keV (contour image, RP2) bands. The coordinates are J2000.0. The subtraction of NXB and the vignetting correction are made. The scales are in the linear (peak to bottom). The red cross shows a peak of the RP2 component. The IC 443 spectrum is taken from the white dashed circle. The bright source in the 5.5-10 keV band at the upper-left is unrelated point source (No. 9 in table 2 of Bocchino \& Bykov 2003).

\subsection{Overview of the RP Process}

In contrast to the well established IP SNR scenario, that of RP SNR has not been established yet. In this paper, we propose a viable scenario based on the standard IP SNR evolution; the RP process is followed after IP process. Figure 2 illustrates the scenario of the spectral evolution of SNRs; how RP is followed after IP.

Phase 1 (IP process): The SNR evolution starts by a shock-heating, in which $k T_{\mathrm{i}}$ and $k T_{\mathrm{e}}$ are nearly 
(a)

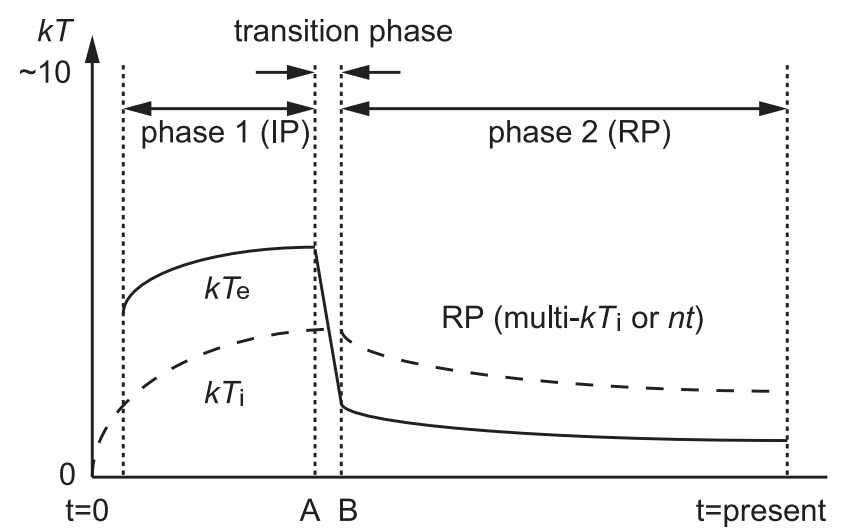

(b)

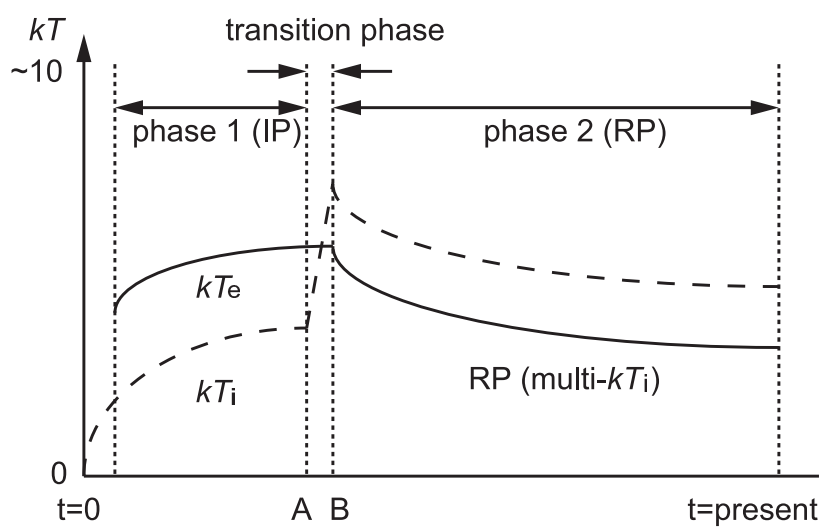

Fig. 2. Schematic pictures of the $k T_{\mathrm{e}}$ and $k T_{\mathrm{i}}$ evolutions in the RP SNR. (a) is the case of electron temperature $\left(k T_{\mathrm{e}}\right)$ decreases, and (b) is the case of ionization temperature $\left(k T_{\mathrm{i}}\right)$ increases.

$0 \mathrm{keV}$. The $k T_{\mathrm{e}}$ quickly increases to several $\mathrm{keV}$, which gradually ionize neutral atoms, and hence $k T_{\mathrm{i}}$ also gradually increases following $k T_{\mathrm{e}}$ (phase 1). Thus phase 1 is ionizing plasma (IP) process of $k T_{\mathrm{e}}>k T_{\mathrm{i}}$. To evolve into the next phase (phase 2) of RP process of $k T_{\mathrm{e}}<k T_{\mathrm{i}}$, there should be a transition phase shown by the epoch $\mathrm{A}$ and $\mathrm{B}$ in figure 2 . In this transition phase, $k T_{\mathrm{e}}$ drops down below $k T_{\mathrm{i}}$, by either conductive cooling by cold cloud (conduction: Kawasaki et al. 2002) or adiabatic cooling by break-out of the plasma in a dense medium to a thin medium (rarefaction; Masai 1994) (figure $2 \mathrm{a}$ ). The other possibility is that $k T_{\mathrm{i}}$ increases by either photo-ionization of an external X-ray source, or ionization by low-energy cosmic rays (LECRs, e.g., Nakashima et al. 2013) (figure 2b) ${ }^{1}$.

Phase 2 (RP process): After the transition, SNR evolution enters RP process, in which free electrons in lower temperature of $k T_{\mathrm{e}}$ are recombining to bound states of ions in higher ionization temperature of $k T_{\mathrm{i}}$. This process makes radiative recombining continuum (RRC). The RRC structure is direct evidence for RP, and has been clearly observed in Si and S in IC 443 (Yamaguchi et al. 2009). As is noted in section 1, the conventional model of the RP SNRs ignores the phase 1 process and treats only the phase 2 process; RP starts from a plasma of $k T_{\mathrm{i}}>k T_{\mathrm{e}}$, where $k T_{\mathrm{i}}$ are all the same in the relevant elements at the epoch $\mathrm{B}$ (here, single-kT $\mathrm{T}_{\mathrm{i}}$ ). Our new model explicitly assumes element dependent $k T_{\mathrm{i}}(z)$ (here, multi-kT $\mathrm{T}_{\mathrm{i}}(\mathrm{z})$ model), which can be smoothly connected to the IP (phase1), well established process for almost all of SNRs.

The multi-kT $\mathrm{T}_{\mathrm{i}}(\mathrm{z})$ model fit has been partially applied to the RP analysis of W28 in the limited energy band (0.6-5 keV) with relevant elements of Ne-Fe (Sawada \& Koyama 2012). For more comprehensive multi-kT $\mathrm{T}_{\mathrm{i}}(\mathrm{z})$ model fit of IC 443 than those of the W28 study, we utilized the wider

\footnotetext{
${ }^{1}$ Depending on the transition process, the duration $(A \rightarrow B)$ is not always short as is given in figure 2. In some cases (LECR origin), its duration is very long extending from the initial to the present epoch.
} 
energy band spectrum of $0.6-10 \mathrm{keV}$. This energy band covers essentially all the relevant elements from $\mathrm{Ne}$ to $\mathrm{Ni}$.

\subsection{Outline of analysis}

The spectrum of the Background (BGD) is extracted from the nearby sky region; the model spectrum is based on Masui et al. (2009), which is consisted of the Milky way halo (MWH), local hot bubble (LHB) and cosmic X-ray background (CXB) (Kushino et al. 2002). For the study of the spectra and fluxes of IC 443 , we use the data within a circle of a 6.5 radius centered on (R.A., Dec. $)_{\text {J2000.0 }}=(6: 17: 10.000,+22: 45: 10.00)$ excluding calibration sources. IC 443 is located at the anticenter region with the Galactic latitude of $b=3^{\circ}$, and hence the Galactic ridge X-ray emission (GRXE) is ignored (Uchiyama et al. 2013; Yamauchi et al. 2016; Koyama 2018). Since we find an Fe I K $\alpha$ line, which should be associated with a continuum, we add a power-law component (PL) with an Fe I $\mathrm{K} \alpha$ line.

Thus the formula of the spectral fitting is the sum of plasma from interstellar medium (ISM) and SNR ejecta (Ejecta), power law ( $P L$ ) plus Fe I K $\alpha$ line (Gaussian (6.4keV)), and nearby X-ray background $(B G D)$. This is given as,

$$
I S M+\text { Ejecta }+P L+\text { Gaussian }(6.4 k e V)+B G D .
$$

The ejecta plasma model (Ejecta) is either a single-k $\mathrm{T}_{\mathrm{i}}$ (conventional model) or multi-kT $\mathrm{k}(\mathrm{z})$ (new model) in the VVRNEI code of the XSPEC package (version 12.9.1). The latter is given as,

$$
\sum_{z=\mathrm{H}}^{\mathrm{z}=\mathrm{Ni}} V V R N E I\left[\text { multi }-k T_{\mathrm{i}}(z)\right],
$$

where $k T_{\mathrm{i}}(z)$ is variable ionization temperature for each element. We added two Gaussians at $\sim 0.8$ $\mathrm{keV}$ and $\sim 1.2 \mathrm{keV}$ to represent the features due to incomplete atomic data for the Fe-L shell complex in the current plasma model (e.g., Nakashima et al. 2013).

As is given in table 1, the observations are extended over large time span. Although the energy resolution in the first observation is not degraded by particle background, those of the latter observations are significantly degraded. In order to compensate this energy resolution variations in the summed spectrum, we apply gsmooth code in XSPEC. The line broadening due to the timedependent variations of the energy resolution are $\sim 30 \mathrm{eV}$ (FWHM). The energy scale is fine-tuned by applying artificial redshift for every element. The gain variation with energy is a concaved function with the amplitude of $+1.4 \% \sim-0.4 \%$ in redshift. 
Model A: At first, we fit the IC 443 spectrum using equation (1) of a single-kT model (RP1) for the ejecta spectrum (Ejecta). Free parameters are $N_{\mathrm{H}}, k T_{\mathrm{e}}, k T_{\mathrm{i}}\left(k T_{\mathrm{i}}(z)\right)$, recombination timescale $\left(n_{\mathrm{e}} t\right)$, normalizations and abundances of $\mathrm{Ne}-\mathrm{Ni}$, while abundances of $\mathrm{He}, \mathrm{C}, \mathrm{N}$, and $\mathrm{O}$ are assumed to be 1 solar. The abundance tables and the atomic data of the lines and continua of the thin thermal plasma are taken from Anders \& Grevesse (1989) and ATOMDB 3.0.7, respectively.

The best fit $k T_{\mathrm{e}}$ of $I S M$ is $\sim 0.2 \mathrm{keV}$, for which there is no significant difference between $\mathrm{CIE}$ and non-equilibrium ionization (NEI). We, therefore, assume that $I S M$ is CIE with the solar abundance (see e.g., Matsumura et al. 2017). The global fit is rejected with a large $\chi^{2} /$ d.o.f. of $2473 / 938$ (2.64).

Model B: Next, we fit with a multi-kT $\mathrm{T}_{\mathrm{i}}(\mathrm{z})$ model (RP1) of equation (2). Free parameters are same as model A. The global fit is significantly improved with $\chi^{2} /$ d.o.f. of 2007/932 (2.15). However, we find significant residuals in the 5-10 keV band, in particular at the Fe I K $\alpha$ (6.4 keV), Fe XXV He $\alpha$ (6.7 keV), Ni VVVII He $\alpha$ (7.8 keV), and at RRC of Fe XXV (8.83 keV).

Model C: We, therefore, add another VVRNEI model (RP2) and a PL component. The VVRNEI model (RP2) is closely related to the $\mathrm{He} \alpha$ and $\mathrm{Ly} \alpha$ lines of $\mathrm{Cr}-\mathrm{Ni}$. The normalization and the recombination timescale for RP2 are linked to those of the lower-temperature component (RP1). The PL is associated with an $\mathrm{Fe} \mathrm{I} \mathrm{K} \alpha$ line. We cannot constrain the photon index $(\Gamma)$ value by the fitting. Non-thermal SNRs typically have $\Gamma$ of 2-3 (e.g., SN1006: Bamba et al. 2003, 2008; RX J1713-3946: Koyama et al. 1997). Accordingly, we assume the power-law index $\Gamma$ to be 2.5. The line width is assumed to be $0 \mathrm{keV}$. The global fit is largely improved with $\chi^{2} /$ d.o.f. of $1674 / 923(1.81)$. In the pure statistical point of view, model $\mathrm{C}$ is still unacceptable. However, possible errors, such as atomic data of Llines of Fe XVI and Fe XVIII, and small calibration errors near the Si I K-edge energy, may not be negligible for the spectrum with very high photon statistics. The actual photon count rate in the Si K-edge band $(1.83-1.85 \mathrm{keV})$ is $(4.20 \pm 0.02) \times 10^{-2}$ counts $\mathrm{s}^{-1}$. Taking account of these systematic errors, we use the model $\mathrm{C}$ results as a reasonable approximation. The best-fit spectra of model $\mathrm{A}, \mathrm{B}$, and $\mathrm{C}$ are given in figure 3, while the best-fit parameters of model A, B, and C are listed in table 2. The $k T_{\mathrm{i}}(z)$ monotonously increases as $z$ in $\mathrm{Ne}-\mathrm{Ca}$, then decreases in $\mathrm{Cr}-\mathrm{Ni}$ for the $\mathrm{RP} 1$ component. The abundances of $\mathrm{Ne}-\mathrm{Ni}$ are generally moderate. Some fractions of the $\mathrm{Cr}-\mathrm{Ni}$ plasma (RP2) show a drastic increase of $k T_{\mathrm{i}}(z)$. In the $\mathrm{Cr}-\mathrm{Ni}$ plasma, the abundance ratio of $\mathrm{Ni} / \mathrm{Fe}$ is $\sim 5$, but the abundances of $\mathrm{Cr}-\mathrm{Ni}$ are very small. 
Table 2. The best-fit parameters of single- $k T_{i}$ (model $\left.A\right)$, multi-k $T_{i}(z)$ (model $B$ ), two-RP of multi-kT $(z)$ (model $\left.C\right)$, and two-RP of multi-k $T_{i}(z)$ in the CC-SNR case (model D).

\begin{tabular}{|c|c|c|c|c|c|c|c|c|c|}
\hline & & \multicolumn{2}{|c|}{ model A } & \multicolumn{4}{|c|}{ model C } & \multicolumn{2}{|c|}{ model D } \\
\hline Component & Parameter & \multicolumn{8}{|c|}{ Values } \\
\hline Absorption & $N_{\mathrm{H}}^{*}$ & \multicolumn{2}{|c|}{$0.65 \pm 0.01$} & \multicolumn{2}{|c|}{$0.65 \pm 0.01$} & \multicolumn{2}{|c|}{$0.67 \pm 0.01$} & \multicolumn{2}{|c|}{$0.67 \pm 0.01$} \\
\hline \multirow[t]{2}{*}{$I S M(\mathrm{CIE})$} & $k T_{\mathrm{e}}^{\dagger}$ & \multicolumn{2}{|c|}{$0.22 \pm 0.01$} & \multicolumn{2}{|c|}{$0.21 \pm 0.01$} & \multicolumn{2}{|c|}{$0.22 \pm 0.01$} & \multicolumn{2}{|c|}{$(=$ model $\mathrm{C})$} \\
\hline & $V E M^{\ddagger}$ & \multicolumn{2}{|c|}{$1.7 \pm 0.7$} & \multicolumn{2}{|c|}{$1.8 \pm 0.1$} & \multicolumn{2}{|c|}{$2.0 \pm 0.1$} & \multicolumn{2}{|c|}{$(=$ model $\mathrm{C})$} \\
\hline \multirow[t]{14}{*}{ Ejecta (RP1) } & $k T_{\mathrm{e}}^{\dagger}$ & \multicolumn{2}{|c|}{$0.53 \pm 0.01$} & \multicolumn{2}{|c|}{$0.53 \pm 0.01$} & \multicolumn{2}{|c|}{$0.56 \pm 0.01$} & \multicolumn{2}{|c|}{$(=$ model $\mathrm{C})$} \\
\hline & $V E M^{\ddagger}$ & \multicolumn{2}{|c|}{$0.47 \pm 0.02$} & \multicolumn{2}{|c|}{$0.54 \pm 0.03$} & \multicolumn{2}{|c|}{$0.46 \pm 0.04$} & \multicolumn{2}{|c|}{$0.042 \pm 0.001$} \\
\hline & $n_{\mathrm{e}} t^{\S}$ & \multicolumn{2}{|c|}{$3.6 \pm 0.1$} & \multicolumn{2}{|c|}{$2.9 \pm 0.2$} & & $=0.1$ & $(=\mathrm{mo}$ & el C) \\
\hline & & $k T_{\mathrm{i}}(z)^{\dagger}$ & $A b^{\|}$ & $k T_{\mathrm{i}}(z)^{\dagger}$ & $A b^{\|}$ & $k T_{\mathrm{i}}(z)^{\dagger}$ & $A b^{\|}$ & $k T_{\mathrm{i}}(z)^{\dagger}$ & $A b^{\|}$ \\
\hline & $\mathrm{Ne}$ & $2.0 \pm 0.1$ & $2.9 \pm 0.3$ & $0.94 \pm 0.12$ & $2.6 \pm 0.2$ & $0.85 \pm 0.05$ & $3.4 \pm 0.5$ & $0.73 \pm 0.02$ & $34 \pm 2$ \\
\hline & $\mathrm{Mg}$ & (link to $\mathrm{Ne}$ ) & $1.8 \pm 0.2$ & $1.1 \pm 0.1$ & $1.5 \pm 0.1$ & $1.1 \pm 0.1$ & $1.6 \pm 0.1$ & (= model $\mathrm{C})$ & $19 \pm 1$ \\
\hline & $\mathrm{Al}$ & (link to $\mathrm{Ne}$ ) & $2.2 \pm 0.3$ & (link to $\mathrm{Mg}$ ) & $1.3 \pm 0.2$ & (link to $\mathrm{Mg}$ ) & $1.1 \pm 0.2$ & (= model C) & $15 \pm 2$ \\
\hline & $\mathrm{Si}$ & (link to $\mathrm{Ne}$ ) & $2.1 \pm 0.2$ & $1.7 \pm 0.1$ & $1.9 \pm 0.1$ & $1.5 \pm 0.1$ & $2.0 \pm 0.2$ & (= model $\mathrm{C})$ & $22 \pm 1$ \\
\hline & $\mathrm{S}$ & (link to $\mathrm{Ne}$ ) & $2.5 \pm 0.2$ & $1.7 \pm 0.1$ & $2.2 \pm 0.1$ & $1.6 \pm 0.1$ & $2.3 \pm 0.2$ & (= model C) & $26 \pm 1$ \\
\hline & $\mathrm{Ar}$ & (link to $\mathrm{Ne}$ ) & $2.2 \pm 0.3$ & $1.9 \pm 0.1$ & $1.8 \pm 0.2$ & $1.6 \pm 0.1$ & $1.9 \pm 0.2$ & $(=$ model $\mathrm{C})$ & $21 \pm 1$ \\
\hline & $\mathrm{Ca}$ & (link to $\mathrm{Ne}$ ) & $3.2 \pm 0.4$ & $3.9 \pm 0.4$ & $0.71 \pm 0.10$ & $2.7 \pm 0.4$ & $0.92 \pm 0.22$ & (= model C) & $10 \pm 1$ \\
\hline & $\mathrm{Cr}=\mathrm{Mn}$ & (link to $\mathrm{Ne}$ ) & $10 \pm 3$ & (link to Fe) & $12 \pm 2$ & (link to Fe) & $<0.4$ & (= model C) & $<8$ \\
\hline & $\mathrm{Fe}$ & (link to $\mathrm{Ne}$ ) & $0.47 \pm 0.29$ & $1.4 \pm 0.1$ & $0.45 \pm 0.02$ & $0.81 \pm 0.09$ & $0.33 \pm 0.03$ & (= model $\mathrm{C})$ & $3.8 \pm 0.2$ \\
\hline & $\mathrm{Ni}$ & (link to $\mathrm{Ne}$ ) & $2.3 \pm 0.2$ & (link to Fe) & $1.7 \pm 0.2$ & (link to Fe) & $2.3 \pm 0.2$ & (= model C) & $28 \pm 2$ \\
\hline Ejecta (RP2) & $k T_{\mathrm{e}}^{\dagger}$ & - & & & & 0.6 & 0.11 & $(=\mathrm{mo}$ & el C) \\
\hline & $V E M^{\ddagger}$ & - & & & & 0.46 & to RP1) & 0.042 & to RP1) \\
\hline & $n_{\mathrm{e}} t^{\S}$ & - & & & & 2.0 (lin & to RP1) & $(=\mathrm{mo}$ & el C) \\
\hline & & & & & & $k T_{\mathrm{i}}^{\dagger}$ & $A b^{\|}$ & $k T_{\mathrm{i}}^{\dagger}$ & $A b^{\|}$ \\
\hline & $\mathrm{Cr}$ & - & - & - & - & (link to Fe) & $0.30 \pm 0.17$ & (link to Fe) & $3.4 \pm 1.6$ \\
\hline & $\mathrm{Mn}$ & - & - & - & - & (link to Fe) & $<0.5$ & (link to Fe) & $<5.0$ \\
\hline & $\mathrm{Fe}$ & - & - & - & - & $6.2 \pm 0.4$ & $0.030 \pm 0.003$ & $(=$ model $\mathrm{C})$ & $0.33 \pm 0.02$ \\
\hline & $\mathrm{Ni}$ & - & - & - & - & (link to Fe) & $0.16 \pm 0.10$ & (link to Fe) & $1.7 \pm 1.0$ \\
\hline$P L$ & $\Gamma^{\sharp}$ & - & & & & 2.5 & xed) & 2.5 & xed) \\
\hline & Norm $^{\sharp}$ & - & & & & 7.8 & $\begin{array}{l}10.1 \\
2.8 \\
\end{array}$ & $(=\mathrm{mo}$ & el C) \\
\hline Gaussian & $E^{* *}$ & - & & & & 6.43 & $=0.04$ & $(=\mathrm{mo}$ & el C) \\
\hline & $F_{6.4 \mathrm{keV}}^{* *}$ & - & & & & & 3.0 & $(=\mathrm{mo}$ & el C) \\
\hline & $\chi^{2} /$ d.o.f. & $2473 / 9$ & $8=2.64$ & $2007 / 9$ & $=2.15$ & $1674 /$ & $3=1.81$ & $1722 / 9$ & $=1.83$ \\
\hline
\end{tabular}

* The unit is $\times 10^{22} \mathrm{~cm}^{-2}$.

$\dagger^{\dagger}$ Units of $k T_{\mathrm{e}}$ and $k T_{\mathrm{i}}(z)$ are $\mathrm{keV}$.

$\ddagger$ Volume emission measure $\left(V E M=n_{\mathrm{e}} n_{\mathrm{H}} V\right)$ with the distance of $1.5 \mathrm{kpc}$. The unit is $10^{58} \mathrm{~cm}^{-3}$.

$\S$ Recombination time scale, where $n_{\mathrm{e}}$ is the electron density $\left(\mathrm{cm}^{-3}\right)$ and $t$ is the elapsed time (s). The unit is $\times 10^{11} \mathrm{~cm}^{-3} \mathrm{~s}$.

II Relative to the solar values in Anders \& Grevesse (1989).

\# Power-law index and flux normalization. The unit of normalization is $10^{-7}$ photons s${ }^{-1} \mathrm{~cm}^{-2} \mathrm{keV}^{-1} \operatorname{arcmin}^{-2}$ at $1 \mathrm{keV}$.

** The Fe I K $\alpha$ line energy and photon flux in units of $\mathrm{keV}$ and $10^{-9}$ photons $\mathrm{s}^{-1} \mathrm{~cm}^{-2} \operatorname{arcmin}^{-2}$, respectively. 

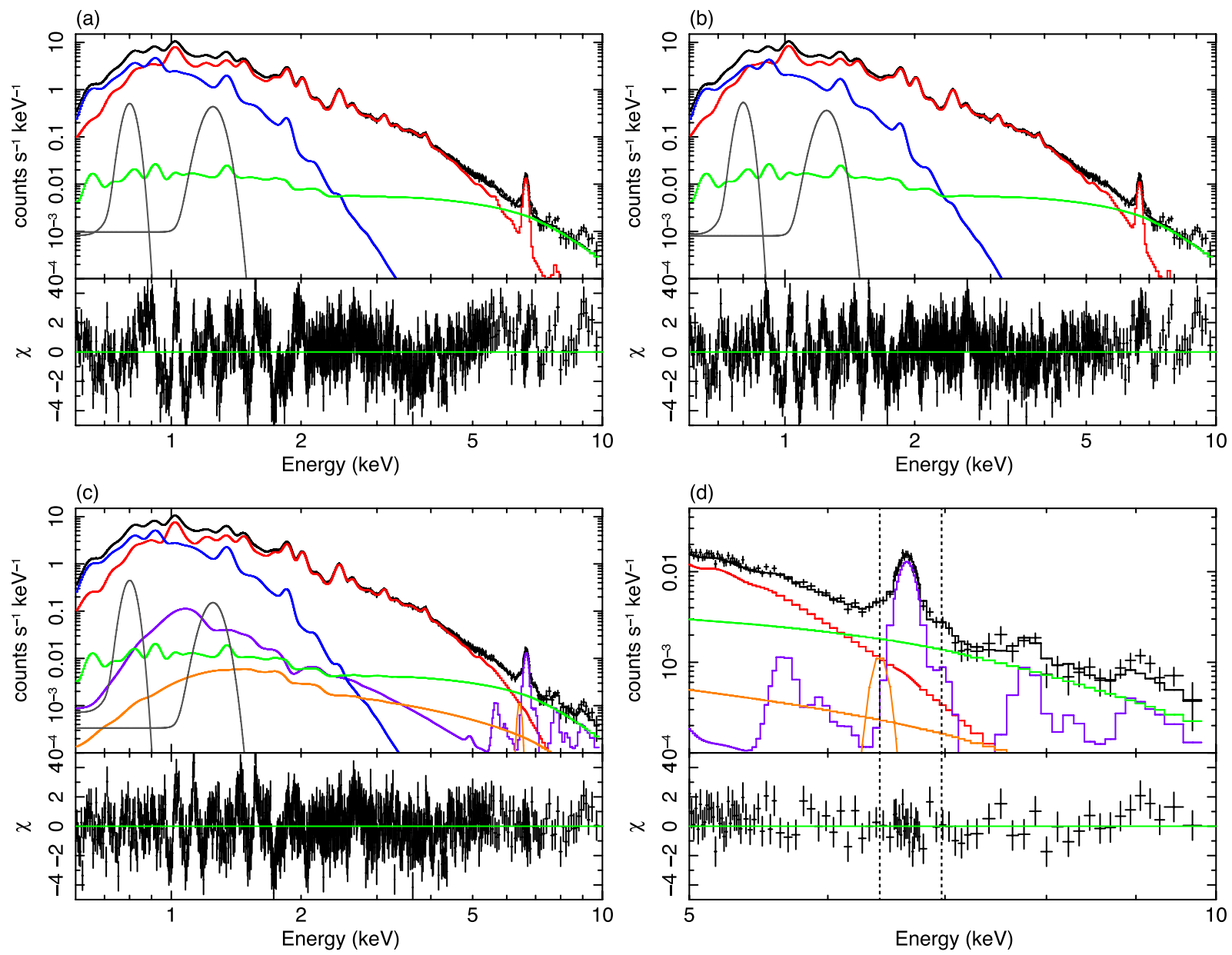

Fig. 3. The XIS spectrum of IC 443 and the best-fit models: (a) single-kT $T_{i}$ (model A), (b) multi-kT $T_{i}(z)($ model $B)$ (c) two-RP of multi-kT $T_{i}(z)$ (model C), and (d) same as (c) but in the 5-10 keV band. The solid red, purple, blue, and orange lines are RP1, RP2, CIE, and PL plus an Fe I K $\alpha$ line, respectively. The gray lines show Fe-L lines, while the green line shows the BGD (=MWH+LHB +CXB). Dotted lines in (d) indicate data excess at the energies of Fe I K $\alpha$ (left) and Fe XXVI Ly $\alpha$ lines (right).

\section{Discussion}

\subsection{Two-RP model and its implication}

The IC 443 spectrum is nicely fitted by a two-RP (RP1 and RP2) of multi-kT $(\mathrm{z})$ model. The best-fit $k T_{\mathrm{e}}$ of RP1 and RP2 are 0.56 and $0.64 \mathrm{keV}$, respectively. Matsumura et al. (2017) also obtained nice fit with a similar two-RP model for IC 443 . However, the best-fit $k T_{\mathrm{e}}$ are 0.24 and $0.61 \mathrm{keV}$, respectively, largely different from our best-fit model. The best-fit abundances are also about two times larger than ours. We note that Matsumura et al. (2017) used limited data set from ID=501006010 (table 1) and limited energy band of $0.6-7.5 \mathrm{keV}$. We, therefore, re-fit their model to our higher quality data (includes all the Obs. ID in table 1) in the wider energy band $(0.6-10 \mathrm{keV})$ to include the RRC of Fe. Then, the fit is rejected with $\chi^{2} /$ d.o.f. $=3589 / 926=3.88$, which is larger than our simpler model A 
of $1-k T_{\mathrm{e}}$ and $1-k T_{\mathrm{i}}$ of $\chi^{2} /$ d.o.f. $=2473 / 938=2.64$. We also compare our best-fit results with the other previous IC 443 report, and find that model $\mathrm{C}$ is the best in reliability and quantity.

In the multi-kT $T_{\mathrm{i}}(\mathrm{z})$ model (model $\mathrm{C}$ ) of ejecta, we found the derived $k T_{\mathrm{i}}(z)$ for each element is not the same, but shows monotonous increase in Ne-Ca and decrease in $\mathrm{Cr}-\mathrm{Ni}$ for RP1 (table 2). Since RP2 has different electron and ionization temperatures from those of RP1, the RP2 plasma may have different origin. The RP2 component shows a higher Ni abundance than Fe (table 2), and might be dominated by the spectrally-hard emission concentrated in the central region of the SNR. Although it is speculative, this hard component emission appears to involve X-ray emission from elongated regions connected to the brightest SNR center (green contours in figure 1) in contrast to the largely smooth distribution of the spectrally-softer RP1 component (dotted contours in figure 1). We speculate that the RP2 component emission might be associated with strong outflows of neutron-rich gas in the deepest core of the CC-SN. Deep follow-up high-resolution X-ray imaging spectroscopic observations of this central region of IC 443 would be required to test this scenario.

In model A-C (table 2), we fit the ejecta plasma with fixed abundances of $\mathrm{He}-\mathrm{O}$ of 1 solar, because these elements do not explicitly appear in the spectrum of $\gtrsim 0.7 \mathrm{keV}$. Since CC-SNRs are largely over-solar abundances of $\mathrm{He}-\mathrm{O}$, we re-fit abundances for the case of $\mathrm{He} \sim 2.6, \mathrm{C} \sim 6.4, \mathrm{~N} \sim 6.5$, and $\mathrm{O} \sim 28$ solar (model D), the mean values of Woosley \& Weaver (1995). The results are given in table 2. The re-fitted abundances in RP1 are about 10 times larger than those of model C. The very small abundances of Fe and $\mathrm{Ni}$ in $\mathrm{RP} 2$ of model $\mathrm{C}$ increase to $\sim 0.3$ and $\sim 1.7$ solar in model $\mathrm{D}$, respectively. The abundances for individual elements and their distribution in the RP1 and RP2 plasma are roughly consistent with the initial assumption of CC-SNR (e.g., Woosley \& Weaver 1995).

From the $k T_{\mathrm{i}}$ given in table 2, the ionization temperature of $\mathrm{Si}$ and $\mathrm{S}$ in IC 443 is estimated to be $\sim 1.5 \mathrm{keV}$ at the start of RP process (epoch A in figure 2). This temperature gives significant Si XIV Ly $\alpha$ and S XVI Ly $\alpha$ as is given in figure 3c. We check the available SNR spectra with similar $k T_{\mathrm{e}}$ of IC $443\left(k T_{\mathrm{e}} \sim 0.3-1 \mathrm{keV}\right)$, but find no significant Si XIV Ly $\alpha$ and S XVI Ly $\alpha$ from the IP SNR samples (e.g., Cygnus loop: Uchida et al. 2011; G272.2-3.2: Kamitsukasa et al. 2016; Kes 79: Sato et al. 2016). As for Fe XXVI Ly $\alpha$, the IP SNR samples are limited, but the good sample is Cas A, which shows no Fe XXVI Ly $\alpha\left(k T_{\mathrm{e}}=1-2 \mathrm{keV}\right.$, Hwang \& Laming 2012). On the other hand, all the spectra of RP SNR samples, which corresponds to the phase 2 (after the transition of IP $\rightarrow$ RP), have clear Si XIV Ly $\alpha$ and S XVI Ly $\alpha$ (e.g., W28: Sawada \& Koyama 2012; G359.1-0.1: Ohnishi et al. 2011). Some RP SNRs with better statistics such as IC 443 (this paper) and W49B (Ozawa et al. 2009) show clear Fe XXVI Ly $\alpha$ line. Since all the RP SNRs would be made by the transition of IP $\rightarrow$ RP (phase $1 \rightarrow$ phase 2), the observed Si XIV Ly $\alpha$, S XVI Ly $\alpha$, and Fe XXVI Ly $\alpha$ in IC 443 should be made by this transition.

The conventional model of electron cooling (figure $2 \mathrm{a}$ ) does not increase the ionization tem- 
perature to make significant Si XIV $\operatorname{Ly} \alpha, \mathrm{S}$ XVI Ly $\alpha$, and Fe XXVI Ly $\alpha$ lines, and hence cannot change the IP to RP in the transition phase. The electron cooling by thermal conduction to cool clouds has another problem that an ionization temperature would also decrease by a contamination of cloud evaporation, and hence the plasma becomes even IP, not RP in the transition phase of figure 2a. Therefore, we rather prefer the model of ionization temperature increase shown in figure $2 b$, in which ionization temperature at the epoch B can be high enough to make Ly $\alpha$ lines of Si, S, and Fe and Fe-RRC. The underlying process would be an irradiation of external X-rays or LECRs.

We discover an Fe I $\mathrm{K} \alpha$ line $(6.4 \mathrm{keV})$ associated with a PL component. In order to explain both the RP and Fe I K $\alpha$ line, we propose a new model which is out of the standard scheme of SNR evolution. LECRs are successively produced by the SNR shock. They irradiate the SNR hot plasma and nearby cold cloud. Fe XXV in the hot plasma is ionized to Fe XXVI, then free electrons with a temperature of $\sim 0.6-0.7 \mathrm{keV}$ in the hot plasma are recombined to the ground state of Fe XXVI after a long recombination process of $(2.0 \pm 0.1) \times 10^{11} \mathrm{~cm}^{-3} \mathrm{~s}$. This process makes the Fe-RRC structure. For the origin of $\mathrm{Fe} \mathrm{I} \mathrm{K} \alpha$ line, one may argue that the origin is irradiation on molecular cloud by an external X-ray source. However this is remote possibility, because no bright X-ray source is found near IC 443. We propose another possibility that the origin of Fe I K $\alpha$ plus continuum is due to LECRs. Our speculation, therefore, is that LECRs are successively produced by the SNR shock, and irradiate both the SNR hot plasma (responsible for RP) and nearby cold cloud (responsible for PL plus Fe I K $\alpha$ ).

\subsection{A possible problem of the RP code}

The model C including VVRNEI in XSPEC, can not fully explain the fluxes of $\mathrm{Fe} X X V \mathrm{He} \alpha$, Fe XXVI Ly $\alpha$ and Fe XXV-RRC in the RP2 spectrum (see 5-10 keV band in figure 3d) in IC 443; the observed flux ratio of $\mathrm{RRC} / \mathrm{He} \alpha$ is larger than code prediction. On the other hand, the RP code of SPEX for the model $\mathrm{C}$ successfully reproduces the $\mathrm{RRC} / \mathrm{He} \alpha$ ratio in the 3.7-10 $\mathrm{keV}$ band spectrum (Ohnishi et al. 2014). We therefore try to re-fit the $5-10 \mathrm{keV}$ band spectrum using the SPEX code of the latest version. Unlike the XSPEC code, this model using the SPEX well reproduces the 5-10 keV band spectrum, in particular the flux ratio of Fe-RRC/Fe XXV He $\alpha$. Thus, we confirm a problem of disagreement between XSPEC and SPEX. To investigate the origin of this code disagreement in the Suzaku spectrum is beyond a scope of this paper. The high resolution calorimeter on board future mission of XRISM may solve this question, because it can resolve the fine structure of $\mathrm{Fe} \mathrm{He} \alpha$ $(x, y, z$, and $w)$ and $\operatorname{Ly} \alpha 1$ and $\operatorname{Ly} \alpha 2$. The absolute flux and the flux ratios of these fine structure lines are essential for the basic atomic process in the RP and the the other plasma processes in SNR. 


\section{Conclusion}

We provide the IC 443 spectrum of the highest signal-to-noise ratio in the widest energy band of $0.6-10 \mathrm{keV}$. The analysis process and results for the unprecedented spectrum are given as follows.

- The multi-kT $\mathrm{i}(\mathrm{z})$ model of RP is nicely fitted to the IC 443 spectrum.

- The distribution of $k T_{\mathrm{i}}(z)$ as $z$ in $\mathrm{Ne}-\mathrm{Ca}$ monotonously increases, then decreases in $\mathrm{Cr}-\mathrm{Ni}$. However, a fraction of $\mathrm{Cr}-\mathrm{Ni}$ show a drastic increase.

- Cr XXIII He $\alpha$ and Fe I K $\alpha$ lines are found for the first time.

- The high abundance ratio of $\mathrm{Ni} / \mathrm{Fe}$ may support that $\mathrm{Ni}$ is over-produced in the neutron rich central region (in the neutron star).

- We interpret that the origin of the RP and an Fe I K $\alpha$ line in IC 443 are enhanced ionization by an irradiation of LECRs.

\section{Acknowledgement}

The authors are grateful to all members of the Suzaku team. This work was supported by the Japan Society for the Promotion of Science (JSPS) KAKENHI Grant Numbers JP16J00548 (KKN), JP15H02090, JP17K14289 (MN) and Nara Women's University Intramural Grant for Project Research (SY).

\section{References}

Anders, E., \& Grevesse, N. 1989, Geochim. Cosmochim. Acta, 53, 197

Bamba, A., Yamazaki, R., Ueno, M., Koyama, K., 2003, ApJ, 589, 827

Bamba, A., et al. 2008, PASJ, 60, S153

Bocchino, F., \& Bykov, A. M. 2003, A\&A, 400, 203

Hwang, U., \& Laming, M. 2012, ApJ, 746, 130

Kamitsukasa, F., Koyama, K., Nakajima, H., Hayashida, K., Mori, K. Katsuda, S., Uchida, H., \& Tsunemi, H. 2016, PASJ, 68, S7

Kawasaki, M. T., Ozaki, M., Nagase, F., Masai, K., Ishida, M., \& Petre, R. 2002, ApJ, 572, 897

Koyama, K., Kinugasa, K., Matsuzaki, K., Nishiuchi, M., Sugizaki, M., Torii, K., Yamauchi, S., \& Aschenbach, B. 1997, PASJ, 49, L7

Koyama, K., et al. 2007, PASJ, 59, S23

Koyama, K. 2018, PASJ, 70, 1

Kushino, A., Ishisaki, Y., Morita, U., Yamasaki, N. Y., Ishida, M., Ohashi, T., \& Ueda, Y. 2002, PASJ, 54, 327 
Masai, K. 1994, ApJ, 437, 770

Masui, K., Mistuda, K., Yamasaki, N. Y., Takei, Y., Kimura, S., \& Yoshino, T. 2009, PASJ, 61, 115

Matsumura, H., Tanaka, T., Uchida, H., Okon, H., \& Tsuru, T. G. 2017, ApJ, 851, 73

Mitsuda, K., et al. 2007, ApJ, 59, S1

Nakashima, S., Nobukawa, M., Uchida, H., Tanaka, T., Tsuru, T. G., Koyama, K., Murakami, H., \& Uchiyama, H. 2013, ApJ, 773, 20

Olbert, C. M., Clearfield, C. R., Williams, N. E., Keohane, J. W., \& Frail, D. A. 2001, ApJ, 554, L205

Ohnishi, T., Koyama, K., Tsuru, T. G., Masai, K., Yamaguchi, H., \& Ozawa, M. 2011, PASJ, 63, 527

Ohnishi, T., Uchida, H., Tsuru, T. G., Koyama, K., Masai K., \& Sawada, M. 2014, ApJ, 784, 74

Ozawa, M., Koyama, K., Yamaguchi, H., Masai, K., \& Tamagawa, T. 2009, ApJ, 706, L71

Petre, R., Szymkowiak, A. E., Seward, F. D., \& Willingale, R. 1988, ApJ, 335, 215

Rho, J., \& Petre, R. 1998, ApJ, 503, L167

Sato, T., Koyama, K., Lee, S.-H., \& Takahashi, T. 2016, PASJ, 68, S8

Sawada, M. \& Koyama, K. 2012, PASJ, 64, 81

Serlemitsos, P. J. et al. 2007, PASJ, 59, 9

Tawa, N., et al. 2008, PASJ, 60, S11

Uchida, H., Tsunemi, H., Tominaga, N., Katsuda, S., Kimura, M., Kosugi, H., Takahashi, H., \& Takakura, S. 2011, PASJ, 63, 199

Uchiyama, H. et al. 2009, PASJ, 61, S9

Uchiyama, H., Nobukawa, M., Tsuru, T. G., \& Koyama, K. 2013, PASJ, 65, 19

Yamaguchi, H., Ozawa, M., Koyama, K., Masai, K., Hiraga, J. S., Ozaki, M., \& Yonetoku, D. 2009, ApJ, 705, 7

Yamauchi, S., Nobukawa, K. K., Nobukawa, M., Uchiyama, H., \& Koyama, K. 2016, PASJ, 68, 59

Welsh, B. Y., \& Sallmen, S. 2003, A\&A, 408, 545

Woosley, S. E., \& Weaver, T. A. 1995, ApJ, 101, 181 